\title{
REDUCTION OF KETONES BY SODIUM \\ BOROHYDRIDE IN THE ABSENCE OF PROTIC \\ SOLVENTS. INTER VERSUS INTRAMOLECULAR MECHANISM
}

\author{
Margaret M. Kayser* and Sonia Eliev \\ Département de chimie, Université de Moncton, Moncton, N.B., Canada \\ odile Eisenstein \\ Department of Chemistry, University of Michigan, Ann Arbor, MI 48109 \\ U.S.A.
}

\section{Abstract:}

Acetone, acetophenone and benzophenone react with sodium borohydride in the absence of protic solvent to give the corresponding tetraalkoxyborates. In view of these results, the possibility of the 4-centre mechanism for these reductions is discusscd.

Sodium borohydride, because of its convenience as well as its efficiency is an attractive and widely used reducing agent. Nonetheless, the mechanism of borohydride reduction of carbonyl function remains the subject of much controversy ${ }^{l}$.

Initially, the reaction was assumed to follow a stepwise hydride ion transfer to the carbonyl carbon atom, resulting in the formation of a tetraalkoxyborate which was subsequently hydrolyzed during the workup procedure to yield alcohol $\underline{I}^{2}$.

\section{$4 \mathrm{R}_{2} \mathrm{CO}+\mathrm{BH}_{4} \Theta \rightarrow \rightarrow\left(\mathrm{R}_{2} \mathrm{CHO}_{4} \mathrm{~B}^{\ominus} \underset{\mathrm{H}_{2} \mathrm{O}}{\longrightarrow} 4 \mathrm{R}_{2} \mathrm{CHOH}\right.$}

The mechanistic studies reported in the following years probed the origin of stereoselectivity ${ }^{3}$, early versus late transition state ${ }^{4}$, solvent and cation influences ${ }^{5}$ and the kinetics of these reactions ${ }^{6}$. The large number of facts and hypotheses amassed over the years served only to cloud the issue and no consensus as to the mechanism of these reactions has been reached.

Recently Wigfield demonstrated that during carbonyl reductions in alcohol solvents, the alkoxyborate anion intermediate contains the solvent alkoxide rather than that of the product and that the ketone is reduced to the free alcohol ${ }^{7}$.

Our ab initio study of $\mathrm{BH}_{4}{ }^{-}$addition to formaldehyde suggested that the nonsynchronous 4-centre transition state may be implicated under the circumstances where the protic solvent is not available ${ }^{8}$. To investigate such a possibility we have studied $\mathrm{NaBH}_{4}$ reductions of acetone, acetophenone and benzophenone in the absence of a protic solvent.

$\mathrm{NaBH}_{4}$ (BDH) was crystallized from boiling dry isopropyl alcohol, dried overnight at $200^{\circ} \mathrm{C}$, and handled under nitrogen. Acetone and acetophenone were dried, purified and distilled. The reduction were carried out neat at 
$0^{\circ} \mathrm{C}$ (acetone) or at room temperature (acetophenone) under nitrogen atmosphere ${ }^{9}$. Benzophenone was crystallized, dissolved in anhydrous THF and treated with $\mathrm{NaBH}_{4}$ under nitrogen, at room temperature for $48 \mathrm{hrs}{ }^{10}$. In all cases the molar ratio of borohydride to ketone was 1 to 4 . The white solid salts obtained were identified as tetraalkoxyborates $\underline{2}, \underline{3}$ and $\underline{4}$ (ir, nmr, mass spectroscopy for 2 ). Acetone and benzophenone were reduced completely - no ketone remained and no free alcohol could be detected. Acetophenone was not entirely reduced after 6 hours at room temperature, some ketone remained. Longer reaction times or higher temperatures gave polymeric products (enolization and polymerization under basic conditions). The salts were subsequently hydrolyzed to yield the corresponding free alcohols.

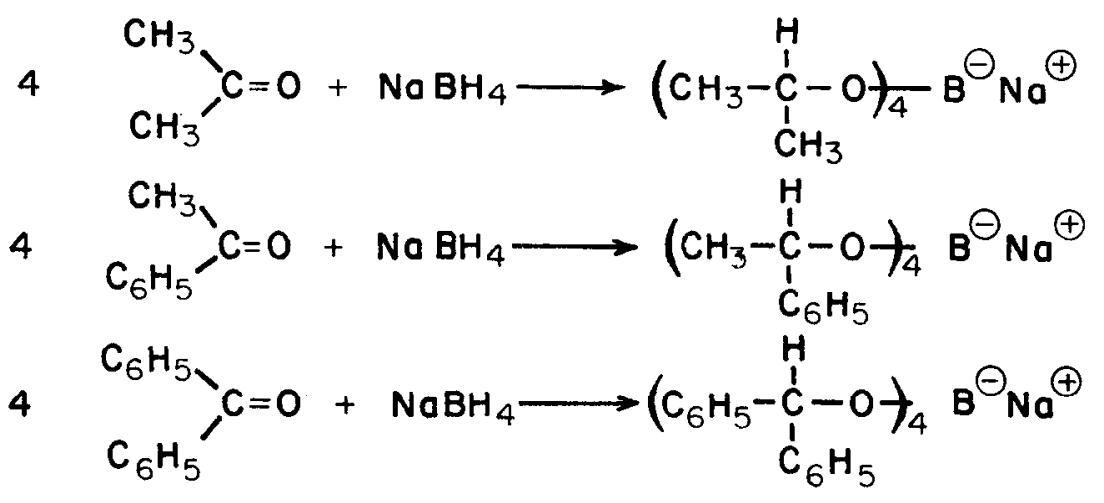

These results suggest that the originally proposed mechanism ${ }^{2}$ is operative in the absence of protic solvents. To rationalize the formation of the tetraalkoxyborate salts two routes may be evoked:

(a) intramolecular transfer of $\mathrm{BH}_{3} 5$ or

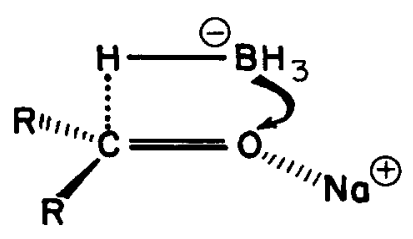


(b) intermolecular transfer of $\mathrm{BH}_{3}$ moiety, as illustrated in 6
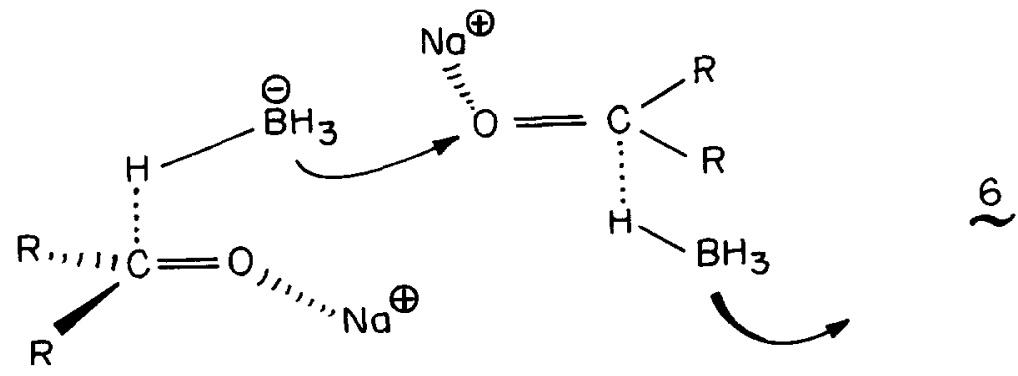

In the former case the $\mathrm{BH}$, transfer may be a true 4-centre transition state (synchronous or non-synchronous) without the participation of another ketone molecule. Alternately $\mathrm{BH}_{3}$ (free or still partly attached to $\mathrm{H}^{-}$) may be stabilized by complexation with the second ketone molecule, acting as a solvent and subsequently be delivered to an alkoxyanion, 7 . Since the binding energy of $\mathrm{H}_{3} \mathrm{~B}---\mathrm{O}=\mathrm{C}_{\mathrm{T}_{\mathrm{H}}}^{\mathrm{H}}$ is weak $(11 \mathrm{kcal} / \mathrm{mol})^{11}$ compared to the binding energy of $\left[\mathrm{H}_{3} \mathrm{~B}-\mathrm{O}-\mathrm{CH}_{3}\right]^{-}(95 \mathrm{kcal} / \mathrm{mol})^{8,12}$ it is quite possible that $\mathrm{BH}_{3}$ will not have an opportunity to lcave the vicinity of the ketonc moleculc where $\mathrm{H}^{-}$has just been transferred. In that case the overall mechanism is nondistinguishable from the true 4-centre transition state.

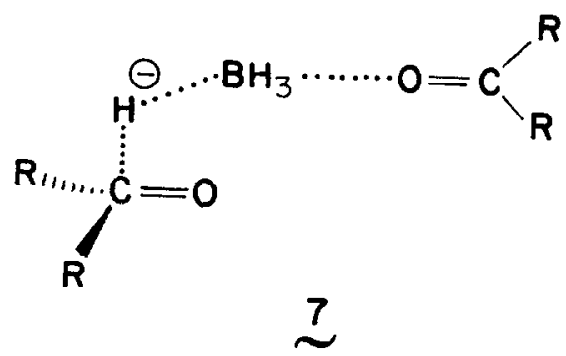

The intermolecular mechanism, involves the simultaneous participation of several molecules and ions; thus for statistical and proximity reasons we tend to believe that the intramolecular transfer is more likely to occur.

\section{ACKNOWLEDGEMENT}

We gratefully acknowledge the support of NATO grant 26-80 (MMK and OE) and the University of Moncton grant (MMK). We would also like to thank the Laboratoire de Chimie Théorique, Centre d'orsay for the use of Monstergauss and computer time.

\section{REFERENCES AND NOTES}

1. D.C. Wigfield, Tetrahedron, 35, 449 (1979).

2. H.C. Brown, Bcranes in O-ganio Chemistry, p. 222: Comeij University Press. 1972, Ithaca and London. 
3. D.H.R. Barton, J. Chem. Soc., 1027 (1953); D.C. Wigfield and D.J. Phelps, J. Org. Chem., 41, 2396 (1976); also see: references 5, 6, 7, 8 cited in reference 8 . O. Eisenstein, H.B. Schlegel and M. Kayser, J. Org. Chem., $\underline{47}, 2886(1982)$.

4. W.G. Dauben, G.J. Fonken, D.S. Noyce, J. Am. Chem. Soc., 75, 6005 (1953); E.C. Ashby, J.T. Laemmle, Chem. Rev., 75, 251 (1976); also see reference 1.

5. H.C. Brown and K.J. Ichikawa, J. Am. Chem. Soc., 83, 4372 (1971); H.C. Brown, E.J. Mead and B.C. Subba Rao, J. Am. Chem. Soc., 98, 5524 (1976).

6. B. Rickborn and M.R. Whesthoff, J. Am. Chem. Soc., 92, 6894 (1970); H.C. Brown, O.H. Wheeler and K. Ichikawa, Tetrahedron, 1, 214 (1957); E.C. Ashby and J.R. Boone, J. Am, Chem. Soc., 98, 786 (1978).

7. D.C. Wigfield and F.W. Gowland, Tetrahedron Lett., 3373, (1973); idem, J. Org. Chem., 42, 1108 (1977); idem, Can. J. Chem., 56, 786 (1978).

8. O. Eisenstein, H.B. Schlegel and M. Kayser, J. Org. Chem., 47, 2886 (1982).

9. The reduction of acetone under this condition was rapid, essentially completed within minutes, while the reduction of acetophenone took several hours. At higher temperatures both acetone and acetophenone polymerized rapidly.

10. Upon evaporation of THF, the solid salt was obtained.

11. The complex formaldehyde- $\mathrm{BH}_{3}$ was calculated at the Hartree-Fock level using a 3-2lG basis set (Monstergauss, version 1976, M. Peterson and R. Poirier, University of Toronto, Department of Chemistry, Toronto, Ontario, Canada; 3-21G basis set: J.S. Binkley, J.A. Pople, W.J. Hehre, J. Am. Chem. Soc., 102, $939(1980)$. Standard geometry was assumed for the formaldehyde part of the complex: J.A. Pople and M. Gordon, J. Am. Chem. Soc., 89, 4253 (1967). B-H distance was $1.19 \mathrm{~A}$ and $\mathrm{H}-\mathrm{B}-\mathrm{H}$ angle $109.47^{\circ}$. The $\mathrm{B}-\mathrm{O}$ distance was optimized $(1.670 \mathrm{~A})$ with a BOC angle kept constant at $120^{\circ}$. The energy of the complex was $-139.47732 \mathrm{a} \cdot \mathrm{u}$.

12. It should be pointed out that the binding energy of the alkoxyanion with $\mathrm{BH}_{3}$ is likely to be exagerated due to the fact that the $\mathrm{Na}^{+}$cation was not taken into account. The cation stabilizes the alkoxy anion and thus diminishes the energy of interaction between $\mathrm{BH}_{3}$ and the anion. The exact value of this effect is difficult to establish as the distance between the oxygen and the $\mathrm{Na}^{+}$cation is unknown.

(Received in USA 9 September 1982) 